\title{
MOVILIZACIÓN, DESMOVILIZACIÓN
}

\author{
Jorge Tárrago Mingo
}

1

"Movilización, desmovilización" es una de las secciones de las que se compone el conocido ensayo El trabajo intelectual. Consejos a los que estudian y a los que escriben escrito por Jean Guitton (Saint Étienne 1901- París 1999) en 1951. Este pequeño libro seguía y completaba a su vez los consejos de otro anterior del mismo autor, Nuevo arte de pensar (1946).

En "Movilización, desmovilización" Guitton recuerda el consejo recibido de un profesor de universidad "que había pasado su vida meditando sobre los métodos del trabajo intelectual". Y cita: "La manera de crear un método - decía- es muy sencilla. Consiste en analizar sistemáticamente la forma en la que habéis trabajado hasta ahora. Hay que examinar, una tras otra y en sus detalles más ínfimos, todas las fases de vuestra actividad intelectual, y esto con un espíritu de severidad inexorable, como si se os pagara muy caro (iy realmente se os paga muy caro!) para descubrir los defectos y sugerir mejoras. Para ello hay que guardarse el amor propio en el bolsillo y saber querer, cosa que se ve poco"l.

El citado profesor había conseguido establecer un método de trabajo personal eliminando la pérdida de tiempo y de esfuerzo después de estudiar cómo Gutenberg, en el caso de la imprenta, había descompuesto en unidades menores -en letras individuales- los bloques completos de grabado de madera que hasta entonces reproducían páginas enteras. Había llegado a la conclusión similar de que se podían descomponer, desmovilizar, los elementos de pensamiento - por ejemplo, de una lectura- clasificarlos en un orden cualquiera -alfabético, cronológico, conceptual-y volverlos a movilizar después, en la medida que fueran útiles multiplicando así su significado y su acción.

1. Nosotros hemos empleado GUITTON, Jean, El trabajo intelectual, RIALP, Madrid, 2010. El capítulo mencionado en pp. 111-112. Y también, GUITTON, J., Nuevo arte de pensar, Encuentro ediciones, Madrid, 2000. 
Con este número diecinueve, Ra, Revista de Arquitectura, se prepara para cumplir veinte años de andadura. De modo quizá inopinado, con un planteamiento más bien fragmentario, los últimos editoriales publicados nos han conducido en su conjunto a reconsiderar la trayectoria de la revista en estas dos décadas. Me refiero especialmente a los que, escritos unas veces por Juan Miguel Ochotorena, yo mismo, o por ambos, desde el número catorce (2012) han tratado de preguntarse por la evolución de las revistas académicas, su vigencia, las particularidades que las distinguen, sus limitaciones, su pertinencia. Serían: "What is the Future of the Academic Publishing?" (Ra14, 2012), "Arquitectura, crítica y géneros literarios" (Ra15, 2013), "Textos de arquitectura” (Ra16, 2014), "El experto y el filósofo" (Ra17, 2015) y "Pensar y hacer los falsos dilemas" (Ra18, 2016).

En efecto, el panorama de las revistas académicas en España ha cambiado radicalmente en la última década. Sin pretender aquí un diagnóstico que se aproxime a una realidad compleja, cabría mencionar cómo al aumento sensible de su número -motivado generalmente por los procesos de acreditación del profesorado en la universidad española- se une una creciente homogeneización en sus procesos y objetivos -a causa de los parecidos procesos de indexación- que, en una visión escueta, probablemente injusta y con alguna excepción, apenas permiten diferenciar hoy unas revistas de otras. Esta es una situación que probablemente ya les trasciende, y de algún modo les obliga.

En su conjunto, e insisto, sin un plan trazado anterior los editoriales mencionados nos han ido confrontando con nuestra trayectoria. Nos han empujado a reconsiderar el modo en el que habíamos trabajado hasta ahora. Y a tomar algunas decisiones que se explicarán a continuación. Y que afectan a la estructura organizativa de la revista y, más importante, a sus contenidos, sobre todo a partir del próximo número $(20,2018)$.

Siguiendo el cauce de estas breves reflexiones, podría pensarse, no sin alguna razón, en que dichas decisiones son fruto de la improvisación. De una cierta fiebre del cambio por el cambio que de cuando en cuando suele afectarnos en el mundo universitario. Porque la revista, podríamos decir que goza de buena salud. ¿Por qué cambiar entonces? ¿No será algo improvisado? Quizá para responder, habría que recurrir a estas palabras de otro francés, Jacques Rancière, que asegura que "improvisar es, en primer lugar, el ejercicio primero de nuestra inteligencia; es la virtud poética, la imposibilidad de decir la verdad, a pesar de sentirla, la que nos hace hablar como poetas, narrar las aventuras de nuestro espíritu y comprobar que son comprendidas por otros aventureros"2.

En fin, con los cambios - algunos ya en marcha y los que se incluirán ya en el número del próximo año- se pretende seguir ahondando en el perfil de revista académica y universitaria, en las razones que originaron su puesta en marcha, la del debate académico de altura, consciente y exigente, en el contexto y panorama actual de las revistas de su tipo.

Entre otros, son los siguientes: desde este número la revista pasa a tener una dirección, un comité de redacción y un equipo editorial. Y a partir del próximo número, al equipo editorial se sumará la figura del editor invitado, procedente siempre de otra Escuela de Arquitectura, institución o centro de investigación. Este es quizá el cambio más significativo, pues el editor invitado se convierte en una figura importante, propondrá el tema sobre el que se hará la llamada a artículos, firmas invitadas y, por tanto, la revista pasaría de un contenido misceláneo a monográfico. El primer editor invitado es Jesús Vassallo, doctor arquitecto por la ETSAM, Master por la Graduate School of Design de Harvard, crítico español, y ahora profesor en la Universidad de Rice en Houston, Texas. El comité científico, por su parte, irá incorporando nuevos miembros progresivamente. La revista, que seguirá siendo anual, pasará a publicar íntegramente los artículos tanto en castellano como en inglés y se revisarán los estándares de diseño actuales proponiendo un nuevo formato.

2. RANCIÈRE, Jacques, El maestro ignorante, Laertes, Barcelona, 2003, p. 87.

Como puede intuirse, y se verá, en el fondo, todo se reduce a desmovilizar para volver a movilizar. 
Además, es también ahora la ocasión de agradecer sinceramente el trabajo de Juan Miguel Ochotorena y José Manuel Pozo en la redacción de la revista. Obsta decir que lo alcanzado se debe en mucho a su tesón y su visión siempre certera y rigurosa. Parecida cosa puede decirse de Carlos Sambricio que deja el comité científico después de un trabajo concienzudo y exigente y que apenas podremos corresponder. Y también es la ocasión para dar la bienvenida a Carlos Labarta Aizpún, entre la Escuela de Ingeniería y Arquitectura de la Universidad de Zaragoza y la ETS de Arquitectura de la Universidad de Navarra, que se une al listado de los que emiten sus juicios y deciden sobre los contenidos de la revista.

\section{EN ESTE NÚMERO}

Félix Solaguren-Beascoa, con motivo del setenta y cinco aniversario de la inauguración de la sede del Ayuntamiento de Aarhus, en "Aarhus: 75 años. Estructura y rigor: la torre, el vestíbulo y las escaleras. La formalización de las escaleras como expresión estructural en Arne Jacobsen" analiza y demuestra con una amplia documentación de archivo, en parte inédita, cómo el requerimiento de la municipalidad al proyecto ganador del concurso, obra de Arne Jacobsen y Erik Møller, de incluir una torre representativa se abordará como un elemento decorativo, y cómo esto marcará el modo de abordar otros elementos significativos en proyectos posteriores de Jacobsen.

Ana Tostoes, en "La fantasía debe ser devuelta a la arquitectura" recupera al arquitecto luso africano Pancho Guedes, su apasionante modo de afrontar la profesión y le presenta como mediador entre el arte y la arquitectura en Mozambique, donde ejerció la mayor parte de su carrera. Y donde creó a su alrededor una importante red de artistas, particularmente con el pintor surrealista Malangatana Ngwenya (1936-2009). El artículo expone con detalle esa otra modernidad alternativa, abierta a las culturas populares y rebosante de fantasía que todavía está por explorar.

Joaquín Medina Warmburg escribe "El mundo como artefacto. Tomás Maldonado en el foco del diseño ambiental (1966-1972)". Recupera y argumenta minuciosamente el papel de liderazgo que el arquitecto uruguayo tuvo, desde Ulm, Princeton y Milán, acerca de los debates sobre el nuevo paradigma acuñado bajo el término de Diseño Ambiental, resultante del giro epistemológico provocado por los enfoques científicos de la ecología, la cibernética o la semiótica en los años sesenta.

Sung-Taeg Nam añade con "El fenómeno de los objetos cotidianos en el periodo de posguerra y el diseño de interiores mediante la elección creativa: Banham, los Smithson y el Arte de habitar" un artículo más a los que con similar temática se han publicado en números anteriores. Aquí, se plantean las reflexiones de Banham sobre la nueva situación de los arquitectos tras la producción masiva de objetos después de la segunda guerra mundial, el concepto de elección creativa, y su interés por el concepto de arte de habitar de los Smithson y los Eames y los objetos cotidianos.

Alberto Ruiz Colmenar presenta en "Las Exposiciones de 1929 en la prensa española no especializada. 'Todo es belleza y paradoja en este pabellón"' la atención sobre estos eventos celebrados en España, en Sevilla y Barcelona, por parte de la prensa generalista y de qué modo ha servido como vehículo de difusión de la arquitectura moderna entre el público no especializado.

Carlos Santamarina-Macho, en "El nuevo nuevo viejo oeste. Un paisaje sin lugar" realiza un recorrido por algunas de las principales reinterpretaciones del mito americano, desde la literatura y las artes de finales del siglo XIX hasta los actuales imaginarios reproducidos por el cine y la televisión. El oeste americano se muestra como un instrumento que sintetiza una determinada interpretación del territorio, la cultura y la sociedad norteamericana, configurando un modo particular de mirar y dotar de sentido a la realidad con la capacidad de adaptarse a diferentes contextos espaciales y temporales. 
Covadonga Lorenzo Cueva demuestra "La influencia de György Kepes en la obra temprana de Juan Navarro Baldeweg realizada en el Center for Advanced Visual Studies del MIT (1971-1975)". Sostiene que ahí se encuentran los principios que han guiado el modo de trabajar de Navarro a lo largo de su vida, como el mismo arquitecto reconoce, y la forma de ver el mundo como un 'paisaje coherente'. El artículo presenta los esquemas, proyectos, piezas, instalaciones e interiores que allí realizó, vinculándolos al contexto en el que se gestaron y mostrando de qué modo parte de su significación plástica respondió a su participación de este entorno colectivo.

David Franco nos propone "Realismo, táctica y espectáculo. Crítica de lo cotidiano en la suburbia de Venturi y Scott Brown" donde examina las ideas que durante los años setenta propusieron Robert Venturi y Denise Scott-Brown en defensa de la suburbia americana. Para ello se traslada la reflexión desde el ámbito de lo simbólico -en que lo enmarcaban sus autores- al de la vida cotidiana, dentro del cual el artículo se centra en la polarización de las prácticas de lo cotidiano - propuesta por Michel De Certeau- entre tácticas y estrategias, así como en las ramificaciones socio-políticas de las mismas.

Héctor García-Diego y María Villanueva analizan exhaustivamente en "Arquitectura, mobiliario y proyecto pedagógico: el pabellón de la Residencia de Señoritas Estudiantes, 1932-1933" la arquitectura y el diseño de mobiliario de esta obra de Carlos Arniches, como factor potenciador del proyecto pedagógico moderno de la Junta para Ampliación de Estudios, y reconstruyen en detalle su proceso de creación a partir de los documentos encontrados en el Archivo de la propia Residencia de Señoritas y de su publicación en las revistas de arquitectura de la época.

Javier Fernández Contreras explica en "Dibujo y repetición: presencia manual de Enric Miralles en la planta del ayuntamiento de Utrecht" la evolución del proyecto desde los cientos de dibujos originales, ilustrando cómo en la repetición y seriación de dicho proceso se produce una incorporación de la peculiar manera de dibujar de Miralles a la geometría de la planta del edificio original.

Jon Arcaraz Puntonet escribe "Superposición: Literal y fenomenal. La influencia de Anni Albers en el gran almacén de Bijenkorf de Marcel Breuer". Sitúa este proyecto como un hito en la trayectoria de Breuer que solo puede ser entendida a través de la influencia de la obra textil de esta artista.

Eduardo Delgado Orusco y Rubén García Rubio documentan el viaje de Louis Kahn a Barcelona con motivo de la invitación a formar parte de las Jornadas Técnicas de la Construcción y el Urbanismo, celebradas en el Recinto de Montjuich del 6 al 9 de junio de 1972. En "La España de Kahn: un viaje y una ensoñación” aprovechan para desvelar el interés del americano por España y la influencia que pudo tener en alguna de sus obras.

Rubén Labiano, describe en "V Bienal de Arquitectura Latinoamericana. BAL2017 en Pamplona" lo acontecido en esta quinta cita que reúne en Pamplona desde 2009 a equipos de muy jóvenes arquitectos de toda Latinoamérica.

Por último, el texto de Mariano Molina "El proyecto de Oskar Hansen para Auschwitz y la monumentalización del debate sobre la guerra". Un tema poco tratado y realmente problemático, la convocatoria del concurso para la creación de un monumento a las víctimas del fascismo en el campo de concentración de Auschwitz-Birkeanu en 1957. El artículo repasa las propuestas finalistas, las estrategias implicadas, los simbolismos y las renuncias. 


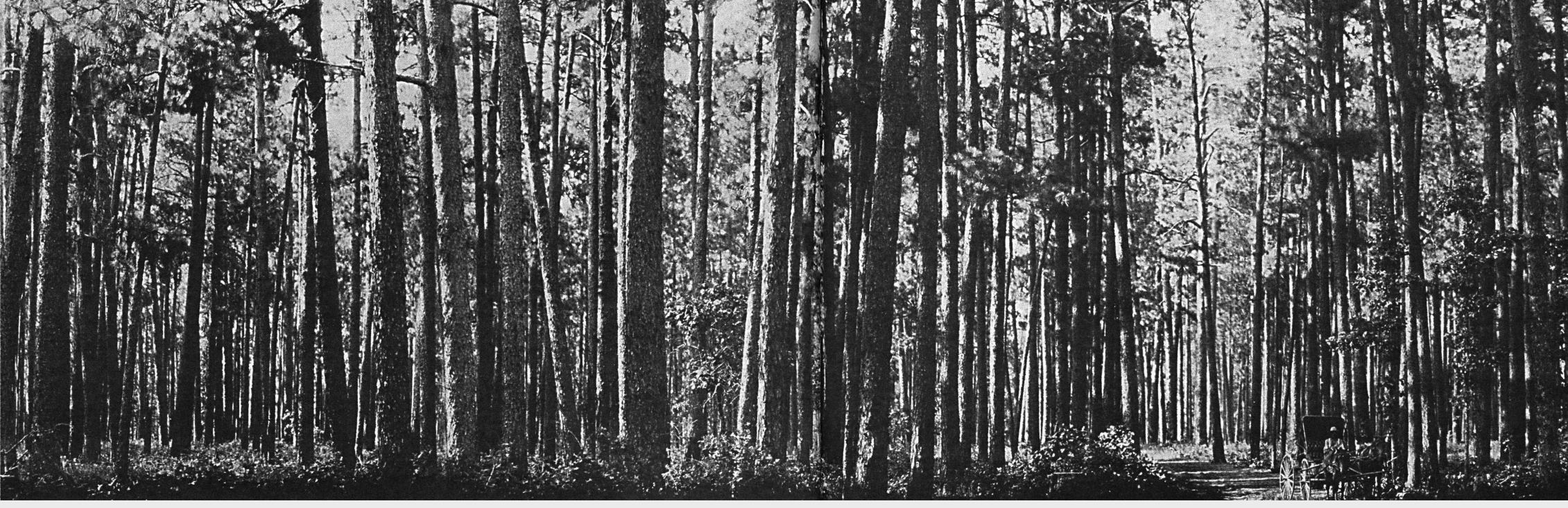

Ra, Revista de Arquitectura Llamada a artículos 2018, Ra 20

\section{La Naturaleza como material de construcción Jesús Vassallo, Editor invitado}

El bosque es por definición la antítesis de la ciudad, un lugar de misterio y sombra donde las leyes de la razón quedan en suspenso. O al menos esa ha sido la narrativa dominante en occidente desde los tiempos de griegos y romanos. Enunciada lúcidamente por Heidegger, la necesidad de abrir un claro en el bosque como acto fundacional y prerrequisito para la formación de cualquier sociedad humana está profundamente enraizada en nuestra conciencia colectiva.

En nuestra época, sin embargo, el supuesto papel del bosque como némesis de la arquitectura y de la civilización que ésta representa ha perdido credibilidad. Sucesivas oleadas de industrialización han revelado la insuficiencia del poder de la naturaleza para poner límites a nuestro apetito, a nuestra capacidad de ocupary consumir el mundo. Hoy nos encontramos en un momento crucial en la historia de la civilización humana, en el que el mayor aumento de población urbana jamás conocido coincide con el reconocimiento de que hemos llegado al límite de nuestra huella sobre el planeta. De esta forma, mientras nos preparamos para alojar a billones de personas en ciudades nuevas y existentes durante las próximas décadas, deberemos necesariamente reconsiderar la relación entre arquitectura y naturaleza.

Al final, todo se reduce a una idea simple pero radical: la necesidad de reconceptualizar la actividad humana en general -la economía es el mejor ejemplo-, y sus productos -lo construido-, como fenómenos internos de la naturaleza. Aunque esta propuesta puede parecer poco intuitiva, hay sin embargo múltiples ejemplos y precedentes históricos de los que podemos valernos, si de verdad pretendemos reconstruir nuestras definiciones de lo natural y lo arquitectónico y producir un nuevo paradigma capaz de impulsarnos en el próximo siglo.
Desde que el poder de la industrialización fue descubierto por primera vez, han surgido periódicamente intentos de repensar la relación de la disciplina de la arquitectura con la naturaleza. Este tema estaba ya presente en el movimiento Arts and Crafts, con su búsqueda de las nuevas formas de la arquitectura en lo natural, e incluso de forma más explícita en la Ciudad Jardín, que reconceptualizó la naturaleza como el principal material de construcción a la escala de la ciudad. También dentro del movimiento moderno, una vez cedieron las primeras fiebres, emergieron ejemplos de esta tendencia: tal vez la Broadacre City de Frank Lloyd Wright sea el más sobresaliente. Los arquitectos nórdicos de la segunda generación también incorporaron la naturaleza de forma integral en su manera de entender la arquitectura, como atestiguan los experimentos que realizaron Arne Jacobsen y Alvar Aalto en sus propias casas, y con sus propias manos. Más cerca de nuestros días, los intentos de Herzog \& de Meuron de construir literalmente con algas o musgo han complicado la distinción entre naturaleza y artificio, prefigurando nuestro interés contemporáneo por los materiales de construcción naturales.

Mientras la construcción en masa con madera transita hacia el éxito comercial y los experimentos con tierra apisonada, adobe o bambú se vuelven más comunes, emerge un camino según el cual la arquitectura algún día crecerá, al menos conceptualmente, de la tierra, en lugar de imponerse sobre ella. La promesa de este camino alberga también el potencial de ayudarnos a repensar la dicotomía con la que enfrentamos los entornos rurales y urbanos, incluso la posibilidad de imaginar y diseñar nuestros ciclos de producción y consumo como parte de un gradiente más amplio de ciclos agrarios y naturales. Con ese fin, esta convocatoria busca temas y ejemplos tanto históricos como contemporáneos, en un amplio abanico de escalas, que puedan contribuir a reconceptualizar la naturaleza como material de construcción o, alternativamente, la arquitectura como vehículo de la naturaleza. Puede que haya llegado la hora de dejar entrar al bosque en nuestras ciudades.
Jesús Vassallo es un arquitecto y crítico español, y actualmente profesor en la Universidad de Rice en Houston, Tejas. Vassallo es Doctor Arquitecto por la ETSAM y Master en Arquitectura por la Graduate School of Design de Harvard. Su trabajo se centra en la interrogación del realismo en arquitectura, a través de la producción de escritos y proyectos. Vassallo es el autor de Seamless: Digital Collage and Dirty Realism in Contemporary Architecture (Park Books, 2016), y actualmente está trabajando en un segundo libro titulado Epics in the Everyday. Sus artículos han sido publicados internacionalmente en revistas como AA Files, $2 G$ Log, Harvard Design Magazine, Future Anterior, Domus, Arquitectura Viva. Desde 2011 es también editor de la revista Circo.

El Editor Invitado y el Comité Científico solicitan contribuciones (véasen las normas editoriales) en inglés o español de arquitectos e investigadores jóvenes y establecidos hasta el 28 de febrero de 2018. A continuación, se mencionan algunos posibles temas a modo de ejemplo. Se valorará la diversidad y el carácter especulativo de las propuestas:

La naturaleza como material de la ciudad (Frank Lloyd Wright: Broadacre City; Ludwig Hilberseimer: The New Regional Pattern, Lafayette Park, Kisho Kurokawa: Agricultural City; Oswald Mathias Ungers: Berlin, A Green Archipelago)

Naturaleza como origen de la forma arquitectónica (Moritz Maurer y Karl Blossfeldt: Lenguaje y ornamento arquitectónico derivado de formas vegetales); Frank Furnes y Louis Sullivan: El Origen del Organicismo Americano; Nicholas Grimshaw: The Eden Project; Toyo Ito Parque de la Gavia)

El arduitecto como jardinero, o el arduitecto en el bosdue (Arne Jacobsen: House and Garden in Soholm, Alvar Aalto Muuratsalo Experimental House, Ralph Erskine: The Box; Alison y Peter Smithson: The Solar Pavilion; Tom Emerson: El Jardín de la ETH; Selgascano: Al Aire).

La naturaleza como bloque de construcción (Herzog \& de Meuron: Ricola-Europe, Bodegas Dominus; Roger Boltshauser: Ozeanium Basel; Anna Herringer: Hostales de Bambú; Peter Zumthor: Pabellón Hannover Pabellón Serpentine; Junya Ishigami: Art Bio Farm, Pabellón en Venecia). 


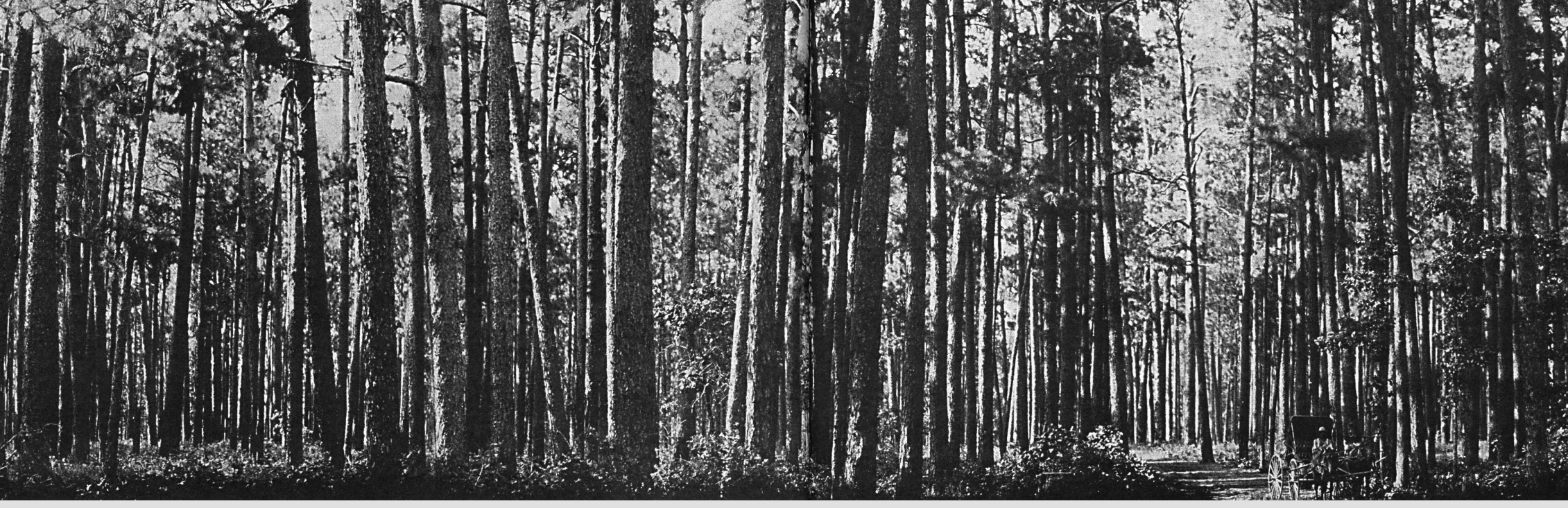

\section{Ra, Revista de Arquitectura Call for papers 2018, Ra 20}

\section{Nature as construction material Jesús Vassallo, guest editor}

The forest is by definition the antithesis of the city, a place of mystery and darkness where the laws of reason do not apply. Or at least, such has been the narrative of western civilization since Greek and Roman times. Best enunciated by Martin Heidegger's famous metaphor, the necessity to clear the forest as a foundational act in order for any form of human society to emerge is deeply engrained in our collective psyche.

Today, the role of the forest as a nemesis of architecture and the civilization for which it stands is however severely weakened. Successive waves of industrialization have rendered the power of nature as insufficient to curb our ambition and appetite to occupy and consume the world. We are now at a pivotal time in the history of human civilization, in which the largest ever movement of population to cities is being paralleled by the realization that we have reached the limit of our footprint on the planet. This entails that as we make preparations to house billions in old and new cities across the globe over the next decades we must necessarily rethink the relationship of architecture and cities to nature.

It all boils down to a simple yet radical idea the necessity to reconsider human activity in general -economy is the best example-, and its products in particular -the built environ ment- as internal rather than external to nature. While this may seem counterintuitive there are however plenty of precedents which we can leverage if we are to reconstruct our cultural definitions of both nature and architecture and produce a new paradigm that can carry us forward through the next century.
Ever since the power of industrialization first revealed itself, there have been periodic bursts or attempts to rethink the relationship of the discipline of architecture to nature. This was explicit in the Arts and Crafts movement, and its search for the new forms of architecture within nature, or even more literally in the City Garden movement, which reconceptualized nature as the main city-building material. Also within the modern movement, once the first fevers of progress started to wear out, instances of this trend emerged, with Frank Lloyd Wright's Broadacre City as perhaps the most salient example at the scale of urbanism. Nordic architects of the second generation of modernism also incorporated nature deep at the core of their approach for architecture, as is evident in the experiments that Alvar Aalto or Arne Jacobsen carried out in their own homes and with their own hands. Closer to our day, the attempts of Herzog \& de Meuron to literally build with algae or moss complicate distinctions between nature and artifice and prefigure our contemporary interest in natural construction materials.

As mass timber construction quickly gravitates towards the mainstream of the construction industry, and experiments with rammed earth, unfired clay, or bamboo become more common, it seems clear that a path emerges in which architecture may one day, at least conceptually, grow out of the soil instead of being imposed on it. This promise also poses the potential to reconsider the divide with which we think about and design rural and urban areas, even the possibility to rethink our cycles of production and consumption as part of a larger gradient of agricultural and natural cycles. This call for papers seeks out both historic and contemporary topics and case studies at a wide range of scales which may contribute to reconceptualize nature as a construction material for architecture, or alternatively, architecture as a vehicle for nature. The time may have come to let the forest back into our cities.
Jesús Vassallo is a Spanish architect and writer, and currently an assistant professor at Rice University in Houston, Texas. He studied architecture at Madrid ETSAM (PhD, Diploma), and at Harvard GSD (MArch II). His work interrogates the problem of realism in architecture through the production of design and scholarship. He is the author of Seamless: Digital Collage and Dirty Realism in Contemporary Arcbitecture (Park Books, 2016) and is currently working on a second manuscript titled Epics in the Everyday. His articles have been published internationally in maga zines such as AA Files, 2 G, Log, Harvard Design Magazine, Future Anterior, Domus, or Arquitectura Viva. Since 2011 he is also an editor of Circo magazine.

The Guest Editor and the Scientific Committee welcome submissions (see author guidelines) in English or Spanish by young and established scholars and architects until February 28, 2018. Here follow a few possible topics by way of example. Diverse and speculative proposals are welcome

Nature as a Material for Building the City (Frank Lloyd Wright: Broadacre City; Ludwig Hilberseimer: The New Regional Pattern, Lafayette Park; Kisho Kurokawa: Agricultural City; Oswald Mathias Ungers Berlin, A Green Archipelago)

Nature as a Source of Architectural Form (Moritz Maurer and Karl Blossfeldt: Nature as origin of architectural language and ornament; Frank Furness and Louis Sullivan: The emergence of American Organicism, Nicholas Grimshaw: The EdenProject; Toyo Ito: Parque de la Gavia)

The Architect as Gardener, or the Architect in the Forest (Arne Jacobsen: House and Garden in Soholm, Alvar Aalto Muuratsalo Experimental House; Ralph Erskine: The Box; Alison and Peter Smithson: The Solar Pavilion; Tom Emerson: The ETH Garden; Selgascano: Between Air).

Nature as a Building Block (Herzog \& de Meuron: Ricola Europe, Dominus Winery; Roger Boltshauser: Ozeanium Basel; Anna Herringer: Bamboo Hostels; Peter Zumthor: Hannover Pavilion, Serpentine Pavilion, Junya Ishigami: Art Bio Farm, Venice Pavilion). 